\title{
Serum Paraoxonase/Arylesterase Activity and Oxidative Stress Status in Children with Metabolic Syndrome
}

\author{
Erdal Eren1, Mahmut Abuhandan2, Abdullah Solmaz2, Abdullah Taşkın3 \\ 1 Harran University, Faculty of Medicine, Department of Pediatric Endocrinology, Şanlıurfa, Turkey \\ 2 Harran University, Faculty of Medicine, Department of Pediatrics, Şanlıurfa, Turkey \\ ${ }^{3}$ Harran University, Faculty of Medicine, Department of Biochemistry, Şanlıurfa, Turkey
}

\begin{abstract}
Objective: This study aimed to measure paraoxonase/arylesterase activities and to evaluate the total oxidant and antioxidant capacities in obese children and in children with metabolic syndrome (MetS).

Methods: A total of 151 children of comparable ages $(13.23 \pm 1.96$ years, $13.45 \pm 1.85$ years and $13.95 \pm 1.31$ years) were enrolled in the study. Forty of these were children with MetS, 55 were obese and 56 were healthy controls. Diagnosis of the MetS was made according to International Diabetes Federation criteria. Paraoxonase/arylesterase activities were evaluated by using paraoxon and phenylacetate substrates. Total oxidant status (TOS) and total antioxidant status (TAS) were measured and oxidative stress index (OSI) was estimated by calculation.

Results: High levels of paraoxonase were detected in the obese group whereas high levels of arylesterase were detected in both MetS and obese groups. Higher values for TOS, TAS and OSI were found in the MetS group ( $\mathrm{p}<0.05)$.

Conclusion: Higher values of mean TOS and OSI in the MetS group than those in the control groups indicate that these parameters may be indicators of future risks such as atherosclerosis in patients with MetS.
\end{abstract}

Key words: Metabolic syndrome, obesity, oxidative stress, paraoxonase

Conflict of interest: None declared

Received: 03.06.2014

Accepted: 07.07.2014

\section{Introduction}

Obesity is a global disease, which can be fatal and which usually has its start in childhood. Metabolic syndrome (MetS), which is encountered in adults, has also been observed in children in recent years. It has been reported that cardiovascular disease risk is higher in children with MetS (1). The abdominal distribution of adipose tissue is an important indicator as intraabdominal adiposity is related to insulin resistance and dyslipidemia and this type of fat distribution is also significant for MetS (2). Hyperglycemia also contributes to the development of lesions in the coronary arteries and aorta (3). In addition, hyperinsulinemia and deteriorated glycemic control are reported to cause an increase in oxidative stress (4). Oxidative stress is also a factor affecting the pathophysiology of MetS $(5,6)$. Paraoxonase (PON)-1 (having PON and arylesterase activities) is an antioxidant enzyme related to high-density lipoproteins (HDL). PON is a glycoprotein synthesized from the liver. Experimental studies indicate that PON prevents atherosclerosis (7). PON1 activity is defined to be low in adults with MetS (8). Studies on adults have established that there is a relationship between coronary heart disease and PON1 activity $(9,10)$. There are a few studies in children on the relationship between PON and MetS. Our aim was to evaluate PON1 activity as well as the total oxidant and antioxidant levels in children.

\footnotetext{
Address for Correspondence

Erdal Eren MD, Uludağ University, Faculty of Medicine, Department of Pediatric Endocrinology, Bursa, Turkey Phone: +90 2242950433 E-mail: dreeren@gmail.com

OJournal of Clinical Research in Pediatric Endocrinology, Published by Galenos Publishing.
} 


\section{Methods}

The study was performed on patients attending the pediatric endocrinology outpatient clinic of our hospital. Patients with body mass index (BMI) values over the 95th percentile for age and gender were accepted as obese (11). Obese cases were divided into two groups as those with MetS and those without MetS. Patients with MetS were defined as subjects showing three or more of the following parameters: abdominal obesity (increased waist circumference, increased waist-height ratio), hypertension, hyperglycemia-insulin resistance, high triglyceride (TG) levels, low HDL levels (12). The control group was composed of healthy age- and gendermatched participants. Subjects with systemic diseases and syndromic diseases were excluded from the study. A total of 151 cases (83 females, 68 males) were included in the study. Data from 40 cases (22 females, 18 males) with MetS, 55 cases ( 27 females, 28 males) with obesity and 56 cases (34 females, 22 males) in the control group were evaluated. Height, weight, BMI and waist circumference/height ratio values of the participants were recorded. Standard deviation scores (SDS) for height, weight and BMI were calculated (13). Informed consent was obtained from the parents of all the children. The study was approved by the Local Ethics Committee of Harran University Medical School.

Blood samples were taken from all cases in the morning between 8:00 and 9:00 a.m. following an overnight fast. The blood samples were centrifuged at $3500 \mathrm{rpm}$ for 10 minutes, then the formed elements were discarded and the serum samples stored at $-80^{\circ} \mathrm{C}$. Measurements included glucose, insulin, alanine transaminase (ALT), plasma TG, total cholesterol, low-density lipoprotein (LDL), HDL, high-sensitivity C-reactive protein (hsCRP) levels, paraoxonase/arylesterase activity, as well as total oxidant and antioxidant capacities. homeostasis model assessment insulin ratioHOMA-IR index (glucose $x$ insulin/405) was used for the detection of insulin resistance. The total antioxidant status (TAS) of plasma was determined using a novel automated measurement method (Abbott Aeroset, Abbott Diagnostics, Abbott Park, IL, USA), developed by Erel (14). In this method, the most potent biological radical, hydroxyl radical, is produced. In the assay, the ferrous ion solution, which is present in reagent 1 [o-dianisidine (10 mM), ferrous ion (45 AM) in the Clark and Lubs solution (75 mM, pH 1.8] is mixed with hydrogen peroxide, which is present in reagent $2 \mathrm{H}_{2} \mathrm{O}_{2}(7.5 \mathrm{mM})$ in the Clark and Lubs solution]. The sequentially produced radicals such as brown-colored dianisidinyl radical cation, formed by the hydroxyl radical, are also potent radicals. Using this method, the antioxidative effect of the sample against the potent free radical reactions initiated by the produced hydroxyl radical is measured. The assay has excellent precision values of lower than $3 \%$. The results were expressed as mmol Trolox Equivalent L-1.

Total oxidant status (TOS) of plasma was determined using a novel automated measurement method, developed by Erel
(15). Oxidants present in the sample oxidized the ferrous iono-dianisidine complex to ferric ion. The oxidation reaction was enhanced by glycerol molecules which were abundantly present in the reaction medium. The ferric ion produced a colored complex with xylenol orange in an acidic medium. The color intensity, which can be measured spectrophotometrically, was related to the total amount of oxidant molecules present in the sample. The assay was calibrated with hydrogen peroxide and the results were expressed in terms of micromolar hydrogen peroxide equivalent per liter ( $\mathrm{mmol} \mathrm{H}_{2} \mathrm{O}_{2}$ Equivalent L-1).

The ratio percentage of TOS level to TAS level gave the oxidative stress index (OSI), an indicator of the degree of oxidative stress (15).

Plasma TG, total cholesterol, LDL and HDL levels were measured by an automated chemistry analyzer (Aeroset, Abbott, USA) using Abbott commercial kits. Serum hsCRP level was measured using an available commercial kit (Roche).

Paraoxonase/arylesterase activities were measured using paraoxon and phenylacetate substrates. The rate of paraoxonhydrolysis (diethyl-p-nitrophenylphosphate) was measured by monitoring the increase of absorbance at $412 \mathrm{~nm}$ at $37^{\circ} \mathrm{C}$. The amount of generated pnitrophenol was calculated from the molar absorptivity coefficient at $\mathrm{pH} 8$, which was 17.000 M-1 cm-1. Paraoxonase activity was expressed as U/L serum. Phenylacetate was used as a substrate to measure the arylesterase activity. Enzymatic activity was calculated from the molar absorptivity coefficient of the produced phenol, $1310 \mathrm{M}-1$ $\mathrm{cm}-1$. One unit of arylesterase activity was defined as $1 \mu \mathrm{mol}$ phenol generated/min under the above conditions and expressed as $U / L$ serum (16). Paraoxonase phenotype distribution was determined by a double substrate method that measures the ratio of paraoxonase activity (with $1 \mathrm{M} \mathrm{NaCl}$ in the assay) to arylesterase activity, using phenylacetate (17).

\section{Statistical Analysis}

The data were analyzed using SPSS (Statistical Package for the Social Sciences, version 11.5 for Windows, SPSS $\mathbb{2}$ Inc, Chicago, IL). The distribution of parametric variables was assessed with one-sample Kolmogorov-Smirnov test when all parametric variables were not distributed normally. The results were presented as mean $\pm S D$. The Kruskal-Wallis and chi-square tests were used for univariate analysis. A two-tailed p-value of less than 0.05 was considered statistically significant.

\section{Results}

Mean ages of the MetS, obese and control groups were $13.23 \pm 1.96$ years (ranging from 9.00 to $16.14 \mathrm{yrs}$ ), $13.45 \pm 1.85$ years (ranging from 9.50 to $6.10 \mathrm{yrs}$ ) and $13.95 \pm 1.31$ years (ranging from 12.03-15.85 yrs), respectively. There was no difference in gender, age, height and height SDS between the groups ( $p>0.05$ ). Weight, weight SDS, BMI, BMI SDS, waist circumference and waist circumference/height ratio of patients in MetS and obese groups were statistically significantly higher 
Eren E et al.

Paraoxonase/Arylesterase in Metabolic Syndrome

than those in the control group ( $p<0.001)$. Auxological data are presented in (Table 1). Weight, weight SDS, BMI, BMI SDS, waist circumference and waist circumference/height ratio values in the MetS group were not statistically significantly different from those in the obese group ( $p>0.05$ ).

Table 2 shows that while there were statistically significant differences in the fasting glucose, fasting insulin, HOMA-IR, ALT, total cholesterol, LDL, HDL, TG, hsCRP, arylesterase, TOS, TAS and OSI values between the MetS and the obese and control groups $(p<0.01)$, there were no statistically significant differences in paraoxonase activity $(p>0.05)$. While there were statistically significant differences in the mean fasting insulin, HOMA-IR, ALT, total cholesterol, hsCRP, paraoxonase/arylesterase activity values between the obese and control groups $(p<0.01)$, there were no statistically significant differences in the mean values of fasting glucose, LDL, HDL, TG, TOS, TAS and OSI ( $p>0.05$ ).

\begin{tabular}{|c|c|c|c|c|}
\hline & MetS $(n=40)$ & Obese $(n=55)$ & Controls $(n=56)$ & $p$-value \\
\hline Age (years) & $13.23 \pm 1.96$ & $13.45 \pm 1.85$ & $13.95+1.31$ & 0.097 \\
\hline Height $(\mathrm{cm})$ & $156.43 \pm 11.02$ & $155.9 \pm 9.07$ & $155.28 \pm 8.44$ & 0.834 \\
\hline Height SDS & $0.12+1.45$ & $-0.03 \pm-0.97$ & $-0.60 \pm 0.88$ & 0.085 \\
\hline Weight (kg) & $82.1 \pm 19.79$ & $77.27 \pm 16.29$ & $46.12 \pm 9.62$ & $<0.001^{a, b}$ \\
\hline Weight SDS & $2.24 \pm 0.57$ & $2.03 \pm 0.59$ & $-0.52 \pm 0.92$ & $<0.001 a, b$ \\
\hline BMI $\left(\mathrm{kg} / \mathrm{m}^{2}\right)$ & $32.99 \pm 6.11$ & $31.29 \pm 4.29$ & $18.85 \pm 2.9$ & $<0.001 a, b$ \\
\hline BMI SDS & $2.24 \pm 0.31$ & $2.13 \pm 0.30$ & $-0.31 \pm 1.06$ & $<0.001 a, b$ \\
\hline Waist circumference $(\mathrm{cm})$ & $100.35 \pm 10.60$ & $97.49 \pm 10.54$ & $65.70 \pm 8.71$ & $<0.001 a, b$ \\
\hline Waist circumference/height & $0.623 \pm 0.063$ & $0.624 \pm 0.052$ & $0.421 \pm 0.053$ & $<0.001 a, b$ \\
\hline
\end{tabular}

Table 2. Laboratory data of the cases in the three groups

\begin{tabular}{|c|c|c|c|c|}
\hline & MetS $(n=40)$ & Obese $(n=55)$ & Controls $(n=56)$ & p-value \\
\hline Fasting glucose (mg/dL) & $94.15 \pm 8.01$ & $90.70 \pm 6.96$ & $88.22 \pm 6.22$ & $0.001^{a}$ \\
\hline Fasting insulin (mU/mL) & $31.02 \pm 14.62$ & $16.73 \pm 9.85$ & $8.43 \pm 4.32$ & $<0.001^{a}, b, c$ \\
\hline HOMA-IR & $7.24 \pm 3.54$ & $3.71 \pm 2.20$ & $1.86 \pm 1.00$ & $<0.001^{a, b, c}$ \\
\hline ALT (IU/mL) & $33.71 \pm 18.63$ & $24.25 \pm 13.42$ & $15.68 \pm 6.44$ & $<0.001 a, b, c$ \\
\hline Total cholesterol (mg/dL) & $182.45 \pm 36.29$ & $154.37 \pm 24.27$ & $139.51 \pm 21.09$ & $<0.001^{a, b, c}$ \\
\hline $\mathrm{LDL}(\mathrm{mg} / \mathrm{dL})$ & $102.65 \pm 37.1$ & $87.16 \pm 22.95$ & $77.97 \pm 16.81$ & $<0.001^{\mathrm{a}}$ \\
\hline $\mathrm{HDL}(\mathrm{mg} / \mathrm{dL})$ & $36.55 \pm 8.05$ & $46.24 \pm 8.29$ & $42.53 \pm 9.50$ & $0.004^{a}, \mathrm{c}$ \\
\hline Triglyceride (mg/dL) & $218.97 \pm 83.17$ & $107.94 \pm 40.38$ & $94.41 \pm 42.73$ & $<0.001^{a, c}$ \\
\hline hsCRP (mg/L) & $0.56 \pm 0.45$ & $0.49 \pm 0.44$ & $0.12 \pm 0.20$ & $<0.001^{a, b}$ \\
\hline Paraoxonase (U/L) & $121.80 \pm 67.90$ & $131.96 \pm 71.07$ & $95.86 \pm 56.15$ & $0.013^{b}$ \\
\hline Arylesterase (U/L) & $104.46 \pm 22.84$ & $98.23 \pm 19.38$ & $83.04 \pm 18.86$ & $<0.001^{a, b}$ \\
\hline $\mathrm{TOS}\left(\mathrm{mmol} \mathrm{H} \mathrm{H}_{2} \mathrm{Eq} / \mathrm{L}\right)$ & $26.77 \pm 12.70$ & $21.55 \pm 7.61$ & $18.89 \pm 5.10$ & $<0.001^{a, c}$ \\
\hline TAS (mmol Trolox Eq/L) & $1.13 \pm 0.14$ & $1.05 \pm 0.16$ & $1.01 \pm 0.15$ & $0.002^{\mathrm{a}}$ \\
\hline OSI (arbitrary unit) & $2.39 \pm 1.13$ & $2.04 \pm 0.64$ & $1.90 \pm 0.64$ & $0.015^{\mathrm{a}}$ \\
\hline
\end{tabular}


Eren $\mathrm{E}$ et al.

Paraoxonase/Arylesterase in Metabolic Syndrome

While there were statistically significant differences in the mean fasting insulin, HOMA-IR, ALT, total cholesterol, HDL, TG and TOS values in the MetS group compared with those in the obese group ( $p<0.01$ ), there were no statistically significant differences in the mean values of fasting glucose, LDL, hsCRP, paraoxonase, arylesterase, TAS and OSI values $(p>0.05)$. Changes in paraoxonase and arylesterase activities of the cases in the three groups are shown in (Figure 1).

\section{Discussion}

Oxidative stress is believed to be the trigger of many diseases. Obesity and/or MetS adversely affect the oxidant system. Low $\mathrm{HDL}$ level, one of the criteria for MetS, is a significant marker of a weak antioxidant defense system. Oxidant effect, which may be caused by LDL, is counteracted by the antioxidant effect of HDL. Moreover, HDL has anti-inflammatory characteristics, so it suppresses cytokine originated endothelial cell adhesion molecules (17). The antioxidant quality of $\mathrm{HDL}$ is related to serum PON1. PON1 activity has been defined to be decreased in adult obese patients (18). Garin et al (8) state that PON1 activity is lower in subjects with MetS than in controls. Tabur et al (19) have reported that there is no difference in PON1 activity between subjects with MetS and obese subjects with no MetS. Studies indicate that PON1 activity decreases with age (20). Studies related to PON and obesity have been performed mostly in adults. However, studies also indicate that PON activity is low in obese children $(21,22)$. Ferre et al $(23)$ have reported that PON1 may play a role in the onset and development of metabolic alterations in childhood obesity. We found elevated PON1 activity in both MetS and obese groups, which was statistically significant between the obese and control groups. Moreover, the arylesterase level, an indicator of PON1 activity, was detected as markedly high both in MetS and obese groups compared with

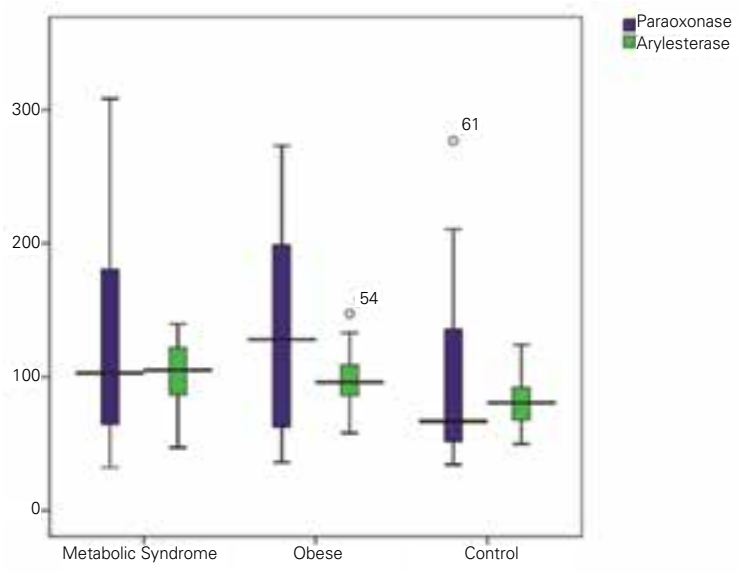

Figure 1. Changes in paraoxonase/arylesterase activity in the three groups the control group. This finding suggests that in children, PON1 prevents lipid peroxidation by playing a preventive role against the oxidative modification of LDL cholesterol. It thus slows the atherosclerosis progression with its antioxidant and antiinflammatory effects. It was also observed that PON1 showed antioxidant characteristics against lipid peroxidation caused by free radicals on cellular membranes and lipoproteins (24).

Lee et al (25) state that antioxidant capacity is related to HDL2 in patients with decreased HDL cholesterol levels when compared with the control groups. These authors report that HDL2-related PON activity increases by nearly three-fold in such patients and that the antioxidant capacity of PON could be related to HDL2 and HDL3. Despite having hypertriglyceridemia and hypercholesterolemia, patients may also have potent PON1 activity and this finding shows that the HDL cholesterol level is not correlated with PON activity. The results of our study were consistent with these findings.

In a study on obese children with and without MetS to evaluate oxidant systems, it has been established that reactive oxidant metabolites are markedly higher in both groups when compared with the control group (26). Our study showed that the fasting insulin level and insulin resistance were significantly high in the MetS and obese groups and that the TOS value in the MetS group was higher than that in both the control and obese groups. Similarly, OSI values were markedly higher in the MetS group. These results indicate that hyperinsulinemia and deteriorated glycemic control contribute to the increase of oxidative stress levels in MetS. Similar to many other diseases, oxidative stress may play a role in the pathogenesis of MetS.

Studies have shown that central obesity is related to the decreased antioxidant capacity in adults. In adult obese cases with or without MetS, TAS levels are lower, while PON and arylesterase levels remain unchanged (19). Another study reveals that children with MetS have high levels of oxidative stress but that the antioxidant stress remains unchanged (27). Pirgon et al (28) state that oxidative stress levels are higher and TAS levels are lower in obese children with nonalcoholic hepatic steatosis, compared with the control group. In contrast, Torun el at (29) found increased TAS levels in obese children. We also found higher TAS levels in the MetS group, a finding which can be evaluated as a potent response of the organism against the oxidant system. Oxidative stress may be an early predictor of atherosclerosis progression. As age increases, the decrease in TAS levels can be effective on atherosclerosis progression. The increased TAS and TOS levels in the MetS group of our study indicate that oxidant stress levels are high in the obese subjects and that the antioxidant system worked to provide the equilibrium. Again, marked increases of PON in the obese group, as well as increased arylesterase levels in both obese and MetS groups are findings which indicate the role of PON1 activity in the antioxidant system. 
CRP is the acute phase reactant, secreted from the liver in reaction to inflammation. It is evident that there are marked increases in CRP and oxidative stress levels in obese children with MetS, and these could pose a risk for heart diseases $(30,31)$. A Norwegian study on 2300 cases with participants between the ages of 9 and 15 years reports a positive correlation between waist circumference and hsCRP (32). While Kitsios et al (33) report that hsCRP levels are higher in obese cases and that the levels are similar in the MetS cases, in another study in Spain, higher hsCRP levels were reported in MetS cases as compared to obese cases (34). In our study, hsCRP levels were found to be significantly higher in the MetS and obese groups compared with the control group. However, there was no difference in hsCRP levels between the MetS and obese groups.

In conclusion, our study showed that oxidant and antioxidant capacities, PON1 activity and hsCRP were high in children with MetS and also in obese children. When compared with studies on adults, the high antioxidant and PON1 levels indicated that protective mechanisms worked. Apparently, the activity of the antioxidant system decreases with age, while the atherosclerotic process is accelerated. Our study also showed that the markers of obesity in humans are initiated during the childhood period.

\section{References}

1. Morrison JA, Friedman LA, Gray-McGuire C. Metabolic syndrome in childhood predicts adult cardiovascular disease 25 years later: the Princeton Lipid Research Clinics Follow-up Study. Pediatrics 2007;120:340-345.

2. Matsuzawa $Y$, Funahashi $T$, Nakamura T. Molecular mechanism of metabolic syndrome $X$ : contribution of adipocytokines adipocyte-derived bioactive substances. Ann N Y Acad Sci 1999;892:146-154.

3. McGill HC Jr, McMahan CA, Malcom GT, Oalmann MC, Strong JP. Relation of glycohemoglobin and adiposity to atherosclerosis in youth. Arterioscler Thromb Vasc Biol 1995; 15:431-440.

4. Holvoet P. Relations between metabolic syndrome, oxidative stress and inflammation and cardiovascular disease. Verh $\mathrm{K}$ Acad Geneeskd Belg 2008;70:193-219.

5. Galassetti P. Inflammation and oxidative stress in obesity, metabolic syndrome and diabetes. Exp Diabetes Res 2012;2012:943706

6. Korkmaz GG, Altınoglu E, Civelek S, Sozer V, Erdenen F, Tabak $\mathrm{O}$, Uzun $\mathrm{H}$. The association of oxidative stress markers with conventional risk factors in the metabolic syndrome. Metabolism 2013;62:828-835. Epub 2013 Feb 11

7. Mackness B, Quarck R, Verreth W, Mackness $M$, Holvoet P. Human paraoxonase-1 overexpression inhibits atherosclerosis in a mouse model of metabolic syndrome. Arterioscler Thromb Vasc Biol 2006;26:1545-1550. Epub 2006 Apr 20

8. Garin MC, Kalix B, Morabia A, James RW. Small, dense lipoprotein particles and reduced paraoxonase- 1 in patients with the metabolic syndrome. J Clin Endocrinol Metab 2005;90:2264-2269. Epub 2005 Feb 1
9. Martinelli N, Girelli D, Olivieri O, Guarini P, Bassi A, Trabetti E, Friso S, Pizzolo F, Bozzini C, Tenuti I, Annarumma L, Schiavon $R$, Franco Pignatti P, Corrocher R. Novel serum paraoxonase activity assays are associated with coronary artery disease. Clin Chem Lab Med 2009;47:432-440.

10. Akçay $A B$, Camsarı $A$, Ozcan T, Ciçek $D$, Akkuş N, Seyis S, Cimen B, Celebi B, Döven O, Cin G. The relationship between paraoxonase-1 activity and coronary artery disease in patients with metabolic syndrome. Turk Kardiyol Dern Ars 2011;39:371-377.

11. Barlow SE; Expert Committee. Expert committee recommendations regarding the prevention, assessment, and treatment of child and adolescent overweight and obesity: summary report. Pediatrics 2007;120(Suppl 4):164192.

12. Alberti KG, Zimmet P, Shaw J. Metabolic syndrome - a new world-wide definition. A consensus statement from the international diabetes federation. Diabet Med 2006;23:469-480.

13. Kuczmarski RJ, Ogden CL, Guo SS, Grummer-Strawn LM, Flegal KM, Mei Z, Wei R, Curtin LR, Roche AF, Johnson CL. 2000 CDC growth charts for the United States: Methods and development. National Center for Health Statistics. Vital Health Stat 11 2002;246:1-190.

14. Erel O. A novel automated method to measure total antioxidant response against potent free radical reactions. Clin Biochem 2004;37:112-119.

15. Erel O. A new automated colorimetric method for measuring total oxidant status. Clin Biochem 2005;38:1103-1111. Epub 2005 Oct 7

16. Haagen L, Brock A. A new automated method for phenotyping arylesterase (EC 3.1.1.2) based upon inhibition of enzymatic hydrolysis of 4-nitrophenyl acetate by phenyl acetate. Eur J Clin Chem Clin Biochem 1992;30:391-395.

17. Eckerson HW, Wyte CM, La Du BN. The human serum paraoxonase/arylesterase polymorphism. Am J Hum Genet 1983;35:1126-1138.

18. Bajnok L, Seres I, Varga Z, Jeges S, Peti A, Karanyi Z, Juhasz A, Csongradi E, Mezosi E, Nagy EV, Paragh G. Relationship of serum resistin level to traits of metabolic syndrome and serum paraoxonase 1 activity in a population with a broad range of body mass index. Exp Clin Endocrinol Diabetes 2008;116:592-599. Epub 2008 May 9

19. Tabur S, Torun AN, Sabuncu T, Turan MN, Celik H, Ocak AR, Aksoy N. Non-diabetic metabolic syndrome and obesity do not affect serum paraoxonase and arylesterase activities but do affect oxidative stress and inflammation. Eur $J$ Endocrinol 2010;162:535-541. Epub 2009 Dec 18

20. Seres I, Paragh G, Deschene E, Fulop T Jr, Khalil A. Study of factors influencing the decreased HDL associated PON1 activity with aging. Exp Gerontol 2004;39:59-66.

21. Koncsos P, Seres I, Harangi M, Páll D, Józsa L, Bajnok $L$, Nagy EV, Paragh G. Favorable effect of short-term lifestyle intervention on human paraoxonase-1 activity and adipokine levels in childhood obesity. J Am Coll Nutr 2011;30:333-339.

22. Seres I, Bajnok L, Harangi M, Sztanek F, Koncsos P, Paragh G. Alteration of PON1 activity in adult and childhood obesity and its relation to adipokine levels. Adv Exp Med Biol 2010;660:129-142

23. Ferré N, Feliu A, García-Heredia A, Marsillach J, París N, Zaragoza-Jordana M, Mackness B, Mackness M, Escribano $\mathrm{J}$, Closa-Monasterolo R, Joven J, Camps J. Impaired paraoxonase-1 status in obese children. Relationships with insülin resistance and metabolic syndrome. Clin Biochem 2013:46:1830-1836. Epub 2013 Sep 10 
Eren $\mathrm{E}$ et al

Paraoxonase/Arylesterase in Metabolic Syndrome

24. Mackness MI, Mackness B, Durrington PN, Connelly PW, Hegele RA. Paraoxonase: biochemistry, genetics and relationship to plasma lipoproteins. Curr Opin Lipido 1996:7:69-76.

25. Lee JH, Park JH, Lee SH, Kim JR, Cho KH. Elevated HDL2paraoxonase and reduced CETP activity are associated with a dramatically lower ratio of LDL-cholesterol/total cholestero in a hypercholesterolemic and hypertriglyceridemic patient. Int J Mol Med 2010;25:945-951.

26. Faienza MF, Francavilla R, Goffredo R, Ventura A, Marzano F Panzarino G, Marinelli G, Cavallo L, Di Bitonto G. Oxidative stress in obesity and metabolic syndrome in children and adolescents. Horm Res Paediatr 2012;78:158-164. Epub 2012 Oct 10

27. Molnár D, Decsi T, Koletzko B. Reduced antioxidant status in obese children with multimetabolic syndrome. Int J Obes Relat Metab Disord 2004;28:1197-1202.

28. Pirgon Ö, Bilgin H, Çekmez F, Kurku H, Dündar BN. Association between insulin resistance and oxidative stress parameters in obese adolescents with non-alcoholic fatty liver disease. J Clin Res Pediatr Endocrinol 2013:5:33-39.

29. Torun E, Gökçe S, Ozgen LT, Aydın S, Cesur Y. Serum paraoxonase activity and oxidative stress and their relationship with obesityrelated metabolic syndrome and non-alcoholic fatty liver disease in obese children and adolescents. J Pediatr Endocrinol Metab 2014;:27:667-675

30. Semiz S, Rota S, Ozdemir O, Ozdemir A, Kaptanoğlu B.Are C-reactive protein and homocysteine cardiovascular risk factors in obese children and adolescents? Pediatr Int 2008;50:419423.

31. Akinci G, Akinci B, Coskun S, Bayindir P, Hekimsoy Z, Ozmen B. Evaluation of markers of inflammation, insulin resistance and endothelial dysfunction in children at risk for overweight. Hormones (Athens) 2008;7:156-162.

32. Steene-Johannessen J, Kolle E, Reseland JE, Anderssen SA, Andersen LB. Waist circumference is related to low-grade inflammation in youth. Int J Pediatr Obes 2010;5:313-319.

33. Kitsios K, Papadopoulou M, Kosta K, Kadoglou N, Papagianni M, Tsiroukidou K. High-sensitivity C-reactive protein levels and metabolic disorders in obese and overweight children and adolescents. J Clin Res Pediatr Endocrinol 2013;5:44-49. Epub 2013 Jul 1

34. Soriano-Guillén L, Hernández-García B, Pita J, DomínguezGarrido N, Del Río-Camacho G, Rovira A. High-sensitivity C-reactive protein is a good marker of cardiovascular risk in obese children and adolescents. Eur J Endocrinol 2008;159:1-4. Epub 2008 May 1 